\title{
Acceptability of Naloxone Co-Prescription Among Primary Care Providers Treating Patients on Long-Term Opioid Therapy for Pain
}

\author{
Emily Behar, MS ${ }^{1,2}$, Christopher Rowe, $\mathrm{MPH}^{7}$, Glenn-Milo Santos, PhD, MPH ${ }^{1,2}$, Diana Coffa, $M D^{2}$, \\ Caitlin Turner, $\mathrm{MPH}^{7}$, Nina C. Santos, $\mathrm{MSPH}^{7}$, and Phillip O. Coffin, MD, MIA ${ }^{1,2}$
}

'San Francisco Department of Public Health, San Francisco, CA, USA; ${ }^{2}$ University of California, San Francisco, San Francisco, CA, USA.

\begin{abstract}
BACKGROUND: Naloxone co-prescription is recommended for patients on long-term opioids for pain, yet there are few data on the practice.

OBJECTIVE: To explore naloxone co-prescribing acceptability among primary care providers for patients on longterm opioids.

DESIGN: We surveyed providers at six safety-net primary care clinics in San Francisco that had initiated naloxone co-prescribing. Providers were encouraged to offer naloxone to patients on long-term opioids or otherwise at risk of witnessing or experiencing an overdose. Surveys were administered electronically 4 to 11 months after coprescribing began.
\end{abstract}

KEY RESULTS: One hundred eleven providers (69\%) responded to the survey, among whom $41.4 \%$ were residents; $40.5 \%$ practiced internal medicine and $55.0 \%$ practiced family medicine. Most (79.3\%) prescribed naloxone, to a mean of 7.7 patients; $99.1 \%$ were likely to prescribe naloxone in the future. Providers reported they were likely to prescribe naloxone to most patients, including those on low doses, defined as $<20$ morphine equivalent $\mathrm{mg}$ daily (59.8\%), $\geq 65$ years old ( $83.9 \%$ ), with no overdose history $(80.7 \%)$, and with no substance use disorder (73.6\%). Most providers felt that prescribing naloxone did not affect their opioid prescribing, $22.5 \%$ felt that they might prescribe fewer opioids, and $3.6 \%$ felt that they might prescribe more. Concerns about providing naloxone were largely administrative, relating to time and pharmacy or payer logistics. Internists (incidence rate ratio $[\mathrm{IRR}]=0.49,95 \% \mathrm{CI}=0.26-0.93, p=0.029$ ), those licensed for 5-20 years (IRR = 2.10, $95 \% \mathrm{CI}=1.35-3.25$, $p=0.001)$, and those with more patients prescribed longterm opioids $(\mathrm{IRR}=1.10,95 \% \mathrm{CI}=1.05-1.14, p<0.001)$ were independently more likely to prescribe a greater number of naloxone compared to participants without these exposures.

CONCLUSIONS: Naloxone co-prescription is considered acceptable among primary care providers. Barriers such as time and dispensing logistics may be alleviated by novel naloxone formulations intended for laypersons recently approved by the U.S. Food and Drug Administration.

Electronic supplementary material The online version of this article (doi:10.1007/s11606-016-3911-z) contains supplementary material, which is available to authorized users.

Received June 15, 2016

Revised September 19, 2016

Accepted October 19, 2016

Published online November 4, 2016
KEY WORDS: opioid analgesics; opioid overdose; naloxone co-prescription; primary care

J Gen Intern Med 32(3):291-5

DOI: $10.1007 / \mathrm{s} 11606-016-3911-\mathrm{z}$

(c) Society of General Internal Medicine 2016

\section{INTRODUCTION}

Drug overdose, driven by opioids, is the leading cause of intentional and unintentional injury-related death in the United States. ${ }^{1}$ Opioid analgesic deaths increased more than fourfold from 2000 to 2014, and heroin-related deaths have increased more than threefold since 2010, with a $26 \%$ increase from 2013 to 2014 . $^{2}$ Opioid overdose, termed an "epidemic" by the Centers for Disease Control and Prevention (CDC), has led to national efforts to address opioid prescribing in clinical care. Recently released CDC guidelines recommend co-prescribing naloxone, the short-acting opioid antagonist used to reverse opioid overdose, to patients on long-term opioid therapy for chronic pain who are on relatively high doses ( $\geq 50$ morphine milligram equivalents per day) or have other high-risk features (e.g. concomitant use of benzodiazepines or substance use disorders). ${ }^{3}$

Naloxone has been distributed to laypersons for use in the event of an overdose since the mid-1990s. Most naloxone services have targeted illicit substance users through lowthreshold community programs like syringe exchanges. This intervention has been associated with both a reduction in the likelihood of death when an overdose occurs and a substantial relative reduction in opioid overdose mortality in communities that distribute the medication. ${ }^{4-6}$

Prescribing naloxone through primary care to patients receiving opioid analgesics, in contrast, has not been well studied. Implementation of a naloxone "co-prescription" program for patients on long-term opioids at the Fort Bragg U.S. Army base was associated with a decline in opioid overdoses from eight to zero per month. ${ }^{7}$ In addition, naloxone co-prescribing in North Carolina was associated with a decrease in the overdose death rate, which was almost exclusively opioid-related, from 46.6 per 100,000 in 2009 to 29.0 per 100,000 in 2010 in the county where it was implemented. ${ }^{8}$ Several studies have suggested that many providers would hypothetically like to prescribe naloxone, yet they also identify barriers to the practice, such as lack of proper training, fear of offending patients, 
and logistical prescribing difficulties. ${ }^{9-13}$ No studies to date have evaluated the actual experience of providers prescribing naloxone to patients.

Naloxone has been available through community distribution programs in San Francisco since 2003, and is associated with marked declines in heroin overdose death. ${ }^{14}$ From 2010 to $2012,92 \%$ of opioid overdose deaths in San Francisco were due to opioid analgesics. ${ }^{15}$ Only $10 \%$ of lay naloxone reversals, however, involved opioid analgesics, suggesting that the population reached with traditional naloxone programming was distinct from those at risk for overdose from opioid analgesics. ${ }^{16}$ In response, the San Francisco Department of Public Health began offering a naloxone prescription to patients on long-term opioids in selected primary care clinics. We surveyed providers at those clinics to evaluate the acceptability of this intervention.

\section{METHODS}

\section{Clinical Intervention}

Six safety-net primary care clinics in San Francisco, accepting only uninsured or publicly insured patients, initiated naloxone co-prescribing in a rolling fashion from February 2013 to April 2014. These clinics were selected from a total of 27 safety-net clinics in San Francisco because they had lost patients to opioid overdose from 2010 to 2012 and maintained a pain management registry (PMR) of patients receiving at least daily opioids for $\geq 3$ months. Patients were eligible for naloxone co-prescription if they were on chronic opioids or otherwise at risk of overdose, including, for example, patients using non-prescription opioids such as heroin. Each clinic received an initial training session at least 1 month prior to project initiation and at least three follow-up sessions focusing on the rationale and indications for prescribing naloxone, available naloxone formulations, insurance coverage information, and communication strategies around discussing naloxone with patients. Training also included instruction for educating patients and caretakers on how to recognize and manage an overdose, including naloxone administration. Emails were sent to providers by study and/or clinic staff during the study period to remind them about naloxone prescribing. Additional details can be found in companion papers. ${ }^{17}$ Study activities were approved by the University of California San Francisco Committee on Human Research (CHR\#13-11168).

\section{Instrument and Data Collection}

The anonymous survey took approximately $5 \mathrm{~min}$ to complete, and was administered online using Qualtrics survey software (Qualtrics LLC, Provo, UT, USA) 4 to 11 months after program rollout from January to December 2014. Clinic staff sent an initial email to providers requesting their participation in the survey; clinic staff sent up to five subsequent follow-up emails reminding staff to complete the survey.
The instrument included 20 questions addressing provider characteristics and naloxone prescribing (Online Appendix 1). Questions addressing provider characteristics included position, medical specialty, number of years licensed to prescribe medications, panel size of patients on opioid analgesics, and patient overdose history. Measures regarding naloxone included number of naloxone prescriptions since initiation of the program, likelihood of prescribing naloxone in the future based on several patient characteristics, impact of naloxone prescribing on opioid prescribing, and barriers to prescribing naloxone. We also assessed nine predefined potential concerns with naloxone co-prescribing derived from relevant literature $^{10}$ and internal discussion among research team clinicians. The survey allowed providers to enter their own concerns and other comments; these were manually categorized and reported if they were not already captured by the predefined concerns. Participating providers were asked to answer all survey questions, regardless of their naloxone prescribing history.

\section{Statistical Analysis}

We assessed bivariate relationships between provider characteristics and both whether the provider had co-prescribed naloxone since the initiation of the clinical program and the number of naloxone prescriptions, using Fisher's exact and Kruskal-Wallis or Wilcoxon rank-sum tests, respectively.

To assess independent correlates of naloxone co-prescribing among providers, we used a negative binomial regression model assessing the number of naloxone prescriptions since initiation of the clinical program. All provider characteristics assessed in bivariable analyses were included as covariates in the primary model, with the exception of provider position, which was excluded due to collinearity with years licensed to prescribe medications $(97.8 \%$ of resident physicians were licensed $<5$ years). The model also included clinic and the time between clinic-specific program initiation and interview date as covariates; the latter was included as an offset. The specification of years licensed to prescribe medications and number of patients prescribed opioid analgesics was determined using orthogonal contrasts to assess linearity across categories; those with no clear evidence $(p>0.05)$ of nonlinearity were converted to continuous variables using the midpoints of each category, and otherwise kept as categorical. In a sensitivity analysis, we used the same negative binomial regression model but included provider position and excluded years licensed to prescribe medications.

\section{RESULTS Provider Characteristics}

Sixty-nine percent (111 of 176) of all providers and $78 \%$ (65 out of 83) of non-resident-level providers completed the survey; interns and resident physicians were less likely to 
complete the survey compared to other providers $\left(\chi^{2}=15.6\right.$, $p<0.001)$. Most providers practiced family or internal medicine. Nearly half had been licensed to practice medicine for less than 5 years. Most saw ten or fewer patients on opioid analgesics per month, and over a quarter had patients who had experienced an opioid overdose in the past year (Table 1). In bivariable analyses, providers' positions $(p=0.001)$, years licensed to prescribe medications $(p=0.015)$, and number of patients prescribed chronic opioids $(p=0.033)$ were significantly associated with prescribing naloxone to patients. The number of naloxone prescriptions since program initiation had a significant crude association with providers' positions (Kruskal-Wallis, $H=28.1, p<0.001)$, years licensed to prescribe medications $(H=19.4, p<0.001)$, number of patients prescribed opioids $(H=37.7, p<0.001)$, and whether providers had patients who had opioid overdoses (Wilcoxon rank-sum $=$ $-2.51, p=0.012$; Table 4).

\section{Acceptability of Naloxone Prescribing}

Most providers $(79.3 \%)$ had prescribed naloxone since program initiation, to a mean of 7.7 patients $(\mathrm{SD}=9.8$; Table 2). Almost all $(99.1 \%)$ reported that they were somewhat to very likely to prescribe naloxone in the future. One provider suggested that naloxone co-prescription had an ancillary benefit of improving the patient-provider relationship: "I expected the decreases in deaths from overdose — but I hadn't thought about how this simple act of prescribing potentially lifesaving treatment has opened up other important conversations that have allowed me to provide better, safer and more compassionate care to my patients." Nearly a quarter $(22.5 \%)$ of providers indicated that prescribing naloxone might lead to prescribing fewer opioids, and $3.6 \%$ suggested that it might lead to prescribing more. Comments indicated that naloxone coprescribing prompted some providers to think more carefully when prescribing opioids for pain: "The conversation about naloxone has changed the dynamic between discussions of harms and benefits for our patients." Most providers showed willingness to prescribe naloxone to various subgroups of patients including those prescribed high- or low-dose opioids (97.7\% and $59.8 \%$, respectively), older patients (83.9\%), and those without any known history of overdose or substance use disorder (80.7\% and $73.6 \%$, respectively; Table 2).

The most frequent concerns were that other staff were needed to teach patients about naloxone, clinic staff often did not assist in teaching patients about naloxone, prescribing naloxone took too much time, and not all insurance covered naloxone. No single concern about prescribing naloxone was endorsed as a "major concern" by more than $10 \%$ of providers (Tables 3 and 4 ).

In the multivariable negative binomial regression model assessing the number of naloxone prescriptions since program initiation, providers practicing family medicine (incidence rate ratio $[\mathrm{IRR}]=0.49,95 \% \mathrm{CI}=0.56-0.93, p=0.028)$ and other specialties ( $\mathrm{IRR}=0.35,95 \% \mathrm{CI}=0.13-0.91, p=0.032$ ) reported fewer naloxone prescriptions compared to those practicing internal medicine. Providers who had been licensed between 5 and 20 years reported more prescriptions compared to those licensed fewer than 5 years $(5-20$ years vs. $<5$ years:

Table 1 Provider Characteristics by Co-Prescription of Naloxone $(n=111)$

\begin{tabular}{|c|c|c|c|c|c|c|c|c|c|c|}
\hline & \multicolumn{2}{|c|}{ All providers } & \multicolumn{2}{|c|}{$\begin{array}{l}\text { Have } \\
\text { co-prescribed } \\
\text { naloxone }\end{array}$} & \multicolumn{2}{|c|}{$\begin{array}{l}\text { Have not } \\
\text { co-prescribed }\end{array}$} & \multicolumn{4}{|c|}{$\begin{array}{l}\text { Number of naloxone co-prescriptions since } \\
\text { program initiation }\end{array}$} \\
\hline & $\mathbf{N}$ & $(\%)^{*}$ & $\mathbf{N}$ & $(\%)^{\dagger}$ & $\mathbf{N}$ & $(\%)^{\dagger}$ & Mean & (SD) & Median & (IQR) \\
\hline Total & 111 & & 88 & $(79.3)$ & 22 & $(19.8)$ & 6.1 & $(9.2)$ & 2 & $(1-6)$ \\
\hline \multicolumn{11}{|l|}{ Position $^{\S \|}$} \\
\hline Resident physician & 46 & $(41.8)$ & 30 & $(34.1)$ & 16 & $(72.7)$ & 1.5 & $(1.6)$ & 1 & $(0-2)$ \\
\hline Other healthcare professional ${ }^{\ddagger}$ & 64 & $(58.2)$ & 58 & $(65.9)$ & 6 & $(27.3)$ & 9.5 & (11.0) & 5 & $(2-10)$ \\
\hline \multicolumn{11}{|l|}{ Specialty } \\
\hline Internal medicine & 45 & $(40.5)$ & 31 & $(35.2)$ & 13 & $(59.1)$ & 6.5 & $(9.4)$ & 2 & $(0-7)$ \\
\hline Family medicine & 61 & $(55.0)$ & 52 & $(59.1)$ & 9 & $(40.9)$ & 6.1 & $(9.5)$ & 2 & $(1-6)$ \\
\hline Other & 5 & $(4.5)$ & 5 & $(5.7)$ & 0 & $(0.0)$ & 3.8 & $(3.6)$ & 2 & $(2-4)$ \\
\hline \multicolumn{11}{|c|}{ Years licensed to prescribe medications ${ }^{\text {}}{ }^{\|}$} \\
\hline$<5$ & 51 & $(45.9)$ & 35 & $(40.2)$ & 16 & $(72.7)$ & 2.8 & $(6.0)$ & 1 & $(0-3)$ \\
\hline $5-20$ & 39 & (35.1) & 36 & (41.4) & 3 & (13.6) & 8.2 & $(9.4)$ & 5.5 & $(3-10)$ \\
\hline$>20$ & 19 & $(17.1)$ & 16 & (18.4) & 3 & $(13.6)$ & 11.4 & $(12.9)$ & 3.5 & $(1-30)$ \\
\hline \multicolumn{11}{|c|}{ Monthly panel of opioid-using patients ${ }^{\S \|}$} \\
\hline $1-5$ & 59 & $(53.2)$ & 40 & $(45.5)$ & 18 & $(81.8)$ & 2 & $(2.4)$ & 1 & $(0-3)$ \\
\hline $6-10$ & 27 & $(24.3)$ & 23 & (26.1) & 4 & (18.2) & 3.6 & $(3.0)$ & 3 & $(2-6)$ \\
\hline $11-15$ & 9 & $(8.1)$ & 9 & $(10.2)$ & 0 & $(0.0)$ & 14.4 & $(10.2)$ & 10 & $(7-20)$ \\
\hline $16-20$ & 7 & $(6.3)$ & 7 & $(8.0)$ & 0 & $(0.0)$ & 19.1 & $(14.0)$ & 30 & $(1-30)$ \\
\hline$>20$ & 9 & $(8.1)$ & 9 & $(10.2)$ & 0 & $(0.0)$ & 20.9 & $(15.6)$ & 20 & $(10-30)$ \\
\hline \multicolumn{11}{|c|}{ Opioid overdoses among patients in past year" } \\
\hline No known opioid overdoses & 78 & $(70.3)$ & 59 & $(67.1)$ & 19 & $(86.4)$ & 4.1 & $(6.0)$ & 2 & $(1-5)$ \\
\hline At least one opioid overdose & 32 & $(28.8)$ & 29 & $(33.0)$ & 3 & (13.6) & 11.2 & (13.3) & 5 & $(1-25)$ \\
\hline
\end{tabular}

*Percentage is column-calculated out of total 111 providers

${ }^{\dagger}$ Percentage is out of column-calculated out of each subcategory of provider

${ }^{7}$ Other healthcare professionals include attending physicians, nurse practitioners, and physician assistants

$s_{p}<0.05$ with Fisher's exact test comparing each characteristic with whether or not the provider had prescribed naloxone

${ }_{p}<0.05$ with Kruskal-Wallis or Wilcoxon rank-sum test comparing each characteristic with the number of naloxone co-prescriptions since program initiation 
Table 2 Provider Acceptability of Naloxone Co-Prescribing $(n=111)$

\begin{tabular}{|c|c|c|}
\hline & $\mathbf{N}$ & $(\%)^{*}$ \\
\hline $\begin{array}{l}\text { Prescribed naloxone to } \geq 1 \text { patient since program } \\
\text { initiation }\end{array}$ & 88 & $(79.3)$ \\
\hline \multicolumn{3}{|l|}{ Likelihood of prescribing naloxone in the future } \\
\hline Not likely & 1 & $(0.9)$ \\
\hline Somewhat likely & 14 & $(12.6$ \\
\hline Moderately likely & 23 & $(20.7)$ \\
\hline Very likely & 71 & $(64.0$ \\
\hline \multicolumn{3}{|c|}{ Somewhat or very likely to prescribe to patient subgroups ${ }^{\dagger}$} \\
\hline On $<20$ morphine equivalent $\mathrm{mg}$ daily & 52 & $(59$. \\
\hline On $>20$ morphine equivalent $\mathrm{mg}$ daily & 86 & $(97.7$ \\
\hline$\geq 65$ years of age & 73 & (83. \\
\hline No known history of overdose & 71 & $\left(800^{\circ}\right.$ \\
\hline No known history of substance use disorder & 64 & (73. \\
\hline \multicolumn{3}{|l|}{ How does prescribing naloxone affect opioid prescribing } \\
\hline No effect & 80 & $(72$. \\
\hline Might prescribe less & 25 & $(22.5)$ \\
\hline Might prescribe more & 4 & (3.6) \\
\hline \multicolumn{3}{|l|}{ Time needed to prescribe naloxone $(\mathrm{min})$} \\
\hline$\leq 5$ & 21 & $(18$. \\
\hline $5-10$ & 44 & \\
\hline $11-15$ & 22 & $(19.8$ \\
\hline$>15$ & 5 & $(4.5)$ \\
\hline Mean number of patients prescribed naloxone ${ }^{\dagger}(\mathrm{SD})$ & 7.7 & $(9.8)$ \\
\hline
\end{tabular}

*Percentages calculated out of all 111 providers; due to missing data, percentages do not add up to $100 \%$ for all questions

${ }^{+}$Number of patients prescribed naloxone was not specified for two providers who had prescribed naloxone, so the mean is calculated among only 86 providers

$\mathrm{IRR}=2.10,95 \% \mathrm{CI}=1.35-3.25, p=0.001 ;>20$ years vs. $<5$ years: $\mathrm{IRR}=0.78,95 \% \mathrm{CI}=0.41-1.49, p=0.452$ ). Providers with more patients receiving opioid analgesics reported more naloxone prescriptions $(\mathrm{IRR}=1.10,95 \% \mathrm{CI}=1.05$ $1.14, p=<0.001)$.

\section{DISCUSSION}

This is the first study to investigate the acceptability of naloxone co-prescription among providers in clinics supporting the practice. Most providers actively engaged in providing naloxone, and the vast majority intended to continue to do so in the future. Providers noted education, pharmacy, and payer
Table 4 Negative Binomial Regression Model Assessing Count of Naloxone Prescriptions $(n=107) *$

\begin{tabular}{lll}
\hline \hline & $\begin{array}{l}\text { IRR (95 \% } \\
\text { CI) }\end{array}$ & $\begin{array}{l}\boldsymbol{p} \\
\text { value }\end{array}$ \\
\hline Specialty & & \\
$\quad$ Internal medicine & Reference & \\
$\quad$ Family medicine & 0.49 & 0.029 \\
$\quad$ Other & 0.35 & 0.031 \\
Years licensed to prescribe medications & Reference & \\
$\quad<5$ & 2.10 & 0.001 \\
$5-20$ & 0.78 & 0.452 \\
$\quad$ Number of patients prescribed opioid & 1.10 & $<0.001$ \\
analgesics & 1.31 & 0.244 \\
$\quad$ Had one or more patients with opioid & & \\
overdose in past year & & \\
\hline
\end{tabular}

*Model adjusted for clinic and time between clinic-specific program initiation and interview date, which was included as an offset $I R R=$ incidence rate ratio

logistics as barriers to naloxone prescribing. Since the time of this study, two formulations of naloxone designed for lay administration (a nasal spray and an auto-injector) have been approved by the U.S. Food and Drug Administration. Unlike other naloxone devices, these new formulations require no assembly and have no additional components such as an atomizer. Additionally, the new formulations are accompanied by instructions for pharmacists. These changes should alleviate most of the concerns noted by providers in this study around education, logistics, and administration.

Providers were willing to prescribe naloxone to most patients, even those likely at low risk of overdose. Providers with 5-20 years of practice or a greater number of patients on opioid analgesic therapy were more likely to prescribe naloxone. These findings may be dependent on the context of the study. Providers were part of a naloxone co-prescribing program in which they were advised to offer naloxone to any patient on long-term opioid therapy; thus, the responses suggest acceptance of the education they had received before the CDC guidelines were issued. ${ }^{3}$ The new CDC naloxone

Table 3 Provider Concerns with Naloxone Co-Prescribing $(n=111)$

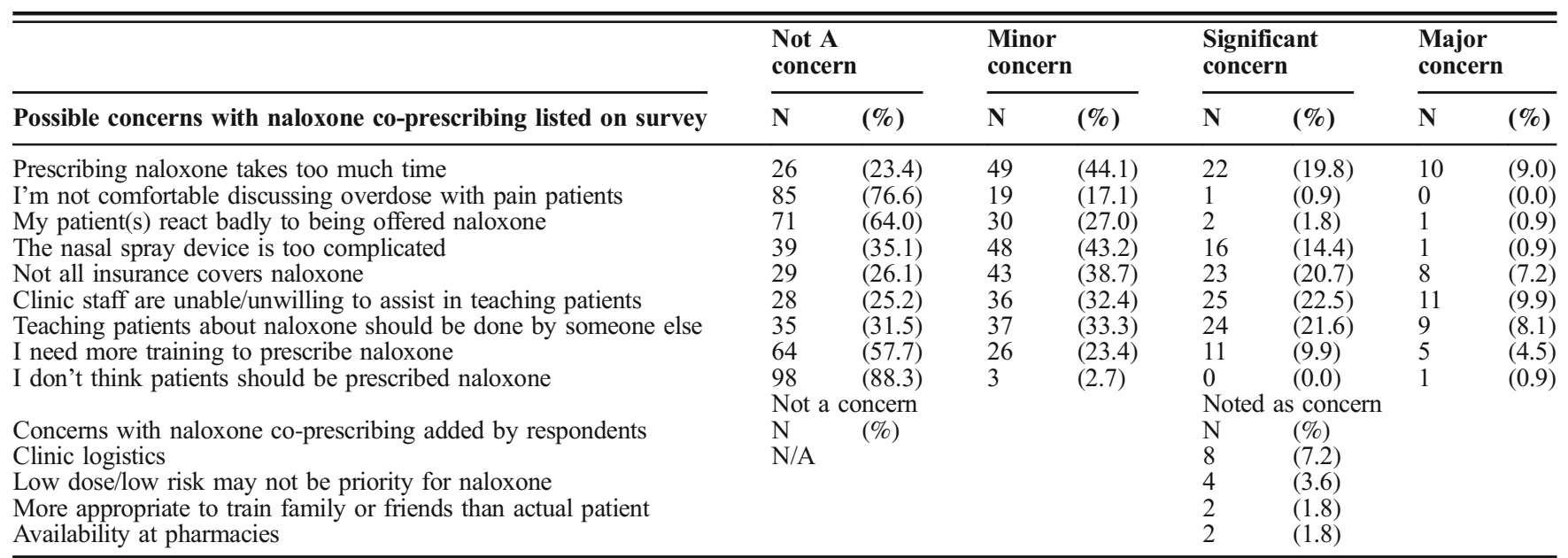


guidelines may serve both to refine provider prescribing practices and to reduce differences in prescribing rates among providers.

Our study has several limitations. First, those who responded to the survey may have been more favorably inclined toward naloxone prescribing than non-respondents. Our response rate, however, was reasonable for a survey, and even higher among non-resident providers, who are likely to be more representative of a clinic culture than are residents. Second, providers in a safety-net clinic may be more inclined to support an intervention such as naloxone prescribing, particularly in San Francisco, where naloxone has been available to laypersons through distribution programs for many years. Generalizability may be further limited by geographic variation in naloxone coverage. Finally, the cross-sectional nature of the survey precludes causal inference. More complicated study designs were not used, since the primary goal of this study was to assess the feasibility and acceptability of naloxone co-prescribing, to lay the groundwork for future intervention efforts.

In conclusion, co-prescribing naloxone to patients on longterm opioid therapy for chronic pain or otherwise at risk of overdose is both feasible and acceptable among primary care providers in safety-net clinics. Barriers to prescribing naloxone are primarily logistical, and may be addressed by recently approved formulations of naloxone intended for lay administration, as well as emerging national guidelines. Nonetheless, to further address these barriers, while maximizing the impact of prescribing naloxone, clinics may benefit by identifying staff to educate patients about naloxone and opioid safety. Future research is needed to address implementation and potential ancillary benefits of co-prescribing naloxone.

\section{Acknowledgements:}

The authors would like to thank Michele Geier and the site leads at each clinic-including Soraya Azari, Barbara Wismer, and Keith Seidel-for participating in the coordination of this project.

Prior Presentations: Some of the data has been the presented at the American Psychiatric Association Conference in Atlanta, Georgia, May 15, 2016, but slides were not shared.

Corresponding Author: Emily Behar, MS; San Francisco Department of Public Health, 25 Van Ness Avenue, Ste. 500, San Francisco, CA 94102, USA (e-mail: Emily.behar@ucsf.edu).

\section{Compliance with Ethical Standards:}

Conflict of Interest: Dr. Phillip O. Coffin has no conflicts directly related to this manuscript; he has led trials that received donated study medications from Alkermes (extended-release naltrexone, through 2015) and Gilead (ledipasvir-sofosbuvir, through present). All other authors declare no conflicts of interest.

Funding Source: National Institutes of Health grant R21DA036776.

\section{REFERENCES}

1. Xu J, Murphy SL, Kochanek KD, Bastian BA. Deaths: Final Data for 2013. Nat Vital Stat Syst. 2016;64(2):1-119.

2. Rudd RA, Aleshire N, Zibbell JE, Gladden RM. Increases in Drug and Opioid Overdose Deaths-United States, 2000-2014. MMWR Morb Mortal Wkly Rep. 2016;64(50-51):1378-1382.

3. Dowell D, Haegerich TM, Chou R. CDC Guideline for Prescribing Opioids for Chronic Pain-United States, 2016. MMWR Recomm Rep. 2016;65(1): 1-49.

4. Strang J. Death matters: understanding heroin/opiate overdose risk and testing potential to prevent deaths. Addiction. 2015;110(Suppl 2):27-35.

5. Walley AY, Xuan Z, Hackman HH, et al. Opioid overdose rates and implementation of overdose education and nasal naloxone distribution in Massachusetts: interrupted time series analysis. BMJ. 2013;346:f174.

6. Mueller SR, Walley AY, Calcaterra SL, Glanz JM, Binswanger IA. A Review of Opioid Overdose Prevention and Naloxone Prescribing: Implications for Translating Community Programming Into Clinical Practice. Subst Abuse. 2015;36(2):240-253.

7. Bartoszek M, Dragovich A, Algeo D, Sellars J. Operation OpioidSAFE: Executive Summary. Womack Army Medical Center: Fort Bragg, NC; 2012.

8. Su A, Brason FW 2nd, Sanford CK, Dasgupta N, Graham J, Lovette B. Project Lazarus: community-based overdose prevention in rural North Carolina. Pain Med. 2011;12(Suppl 2):S77-S85.

9. Beletsky L, Ruthazer R, Macalino GE, Rich JD, Tan L, Burris S. Physicians' knowledge of and willingness to prescribe naloxone to reverse accidental opiate overdose: challenges and opportunities. J Urban Health. 2007;84(1): 126-136.

10. Binswanger IA, Koester S, Mueller SR, Gardner EM, Goddard K, Glanz JM. Overdose Education and Naloxone for Patients Prescribed Opioids in Primary Care: A Qualitative Study of Primary Care Staff. J Gen Intern Med. 2015;30(12):1837-1844.

11. Leece $\mathbf{P}$, Orkin A, Shahin RM, Steele L. Can naloxone prescription and overdose training for opioid users work in family practice? Can Fam Physician. 2015;61(6):538-543.

12. Matheson C, Pflanz-Sinclair C, Aucott L, et al. Reducing drug related deaths: a pre-implementation assessment of knowledge, barriers and enablers for naloxone distribution through general practice. BMC Fam Pract. 2014;15: 12 .

13. Wilson JD, Spicyn N, Matson P, Alvanzo A, Feldman L. Internal medicine resident knowledge, attitudes, and barriers to naloxone prescription in hopsital and clinic settings. Subst Abus. 2016;37(3):480-487.

14. Rowe C, Santos GM, Vittinghoff E, Wheeler E, Davidson P, Coffin PO. Predictors of participant engagement and naloxone utilization in a community-based naloxone distribution program. Addiction. 2015;110(8):1301-1310.

15. Visconti AJ, Santos GM, Lemos NP, Burke C, Coffin PO. Opioid Overdose Deaths in the City and County of San Francisco: Prevalence, Distribution, and Disparities. J Urban Health. 2015;92(4):758-772.

16. Rowe C, Santos GM, Vittinghoff E, Wheeler E, Davidson P, Coffin PO. Neighborhood-Level and Spatial Characteristics Associated with Lay Naloxone Reversal Events and Opioid Overdose Deaths. J Urban Health. 2016;93(1): 117-130.

17. Coffin PO, Behar E, Rowe C, et al. Non-randomized intervention study of naloxone co-prescription for primary care patients on long-term opioid therapy for pain. Ann Intern Med 2016;165(4):245-252. 